\title{
On solution of total least squares problems with multiple right-hand sides
}

\author{
Iveta Hnětynková ${ }^{1,2, *}$, Martin Plešinger ${ }^{2,3, * *}$, and Zdeněk Strakoš ${ }^{1,2, * * *}$ \\ ${ }^{1}$ Charles University in Prague, Faculty of Mathematics and Physics, Sokolovská 83, 18675 Prague 8, Czech Republic \\ ${ }^{2}$ Institute of Computer Science, Academy of Sciences of the Czech Republic \\ ${ }^{3}$ Technical University of Liberec, Faculty of Mechatronics and Interdisciplinary Engineering Studies, Czech Republic
}

\begin{abstract}
Consider a linear approximation problem $A X \approx B$ with multiple right-hand sides. When errors in the data are confirmed both to $B$ and $A$, the total least squares (TLS) concept is used to solve this problem. Contrary to the standard least squares approximation problem, a solution of the TLS problem may not exist. For a single (vector) right-hand side, the classical theory has been developed by G. H. Golub, C. F. Van Loan [2], and S. Van Huffel, J. Vandewalle [4], and then complemented recently by the core problem approach of C. C. Paige, Z. Strakoš [5-7]. Analysis of the problem with multiple right-hand sides is still under development. In this short contribution we present conditions for the existence of a TLS solution.
\end{abstract}

Copyright line will be provided by the publishe

\section{Introduction}

Consider an orthogonally invariant linear approximation problem with $d$ right-hand sides

$$
A X \approx B, \quad A \in \mathbb{R}^{m \times n}, \quad X \in \mathbb{R}^{n \times d}, \quad B \in \mathbb{R}^{m \times d},
$$

where the uninteresting case is excluded by the assumption $A^{T} B \neq 0$. With no loss of generality assume $m \geq n+d$ (add zero rows if necessary). In total least squares (TLS) $[1,2,4]$ this problem is solved by constructing minimal correction to $B$ and $A$ such that the corrected system is compatible,

$$
\min _{E, G, X}\|[E, G]\|_{F} \quad \text { subject to } \quad(A+E) X=B+G .
$$

With $d=1$ the problem (1) reduces to $A x \approx b$, where $b$ is an $m$-vector. This problem has been analyzed in [2,4] and then revised in [5-7], see also [3]. In [5-7], it is shown how the necessary and sufficient information for solving (1) can be extracted from the data using the so called core reduction. With the singular value decomposition (SVD)

$$
[b, A]=U \Sigma V^{T}=\sum_{i=1}^{n+1} u_{i} \sigma_{i} v_{i}^{T}, \quad \sigma_{1} \geq \cdots \geq \sigma_{p}>\sigma_{p+1}=\cdots=\sigma_{n+1} \geq 0,
$$

and the corresponding partitioning

$$
V=\left[\begin{array}{l|l}
v_{11} & v_{12} \\
\hline V_{21} & V_{22}
\end{array}\right], \quad v_{11} \in \mathbb{R}^{1 \times p}, v_{12} \in \mathbb{R}^{1 \times(n-p+1)}, V_{21} \in \mathbb{R}^{n \times p}, V_{22} \in \mathbb{R}^{n \times(n-p+1)},
$$

the classical analysis gives the following conditions on existence and uniqueness of a TLS solution in the case $d=1$, see [7] for more details, and the relationship to the core problem theory, which in particular gives an insight into the non-existence of the TLS solution:

- If $v_{12} \neq 0$ with $p=n$, i.e. $\sigma_{n+1}$ is simple and the corresponding right singular vector of $[b, A]$ has a nonzero first component, then there exists the unique TLS solution of (1).

- If $v_{12} \neq 0$ with $p<n$, i.e. $\sigma_{n+1}$ is multiple and there exists right singular vector corresponding to $\sigma_{n+1}$ with a nonzero first component, then the TLS problem (2) does not have a unique solution. The unique minimum norm solution can be constructed.

- If $v_{12}=0$, i.e. all right singular vectors corresponding to $\sigma_{n+1}$ have zero first components, then the TLS solution of (1) does not exist.

* Corresponding author E-mail: iveta.hnetynkova@mff.cuni.cz, Phone: +420221913362 Fax: +42022481 1036. Part of this work was performed during the academic year 2007/2008 while visiting Arizona State University, Tempe, AZ, U.S.A.

** e-mail: mata@cs.cas.cz

*** e-mail: strakos@cs.cas.cz 


\section{Classification of TLS problems}

In order to handle a possible multiplicity of $\sigma_{n+1}$ in case $d>1$, consider the SVD

$$
\begin{gathered}
{[B, A]=U \Sigma V^{T}=\sum_{i=1}^{n+d} u_{i} \sigma_{i} v_{i}^{T},} \\
\sigma_{1} \geq \ldots \geq \sigma_{p}>\sigma_{p+1}=\ldots=\sigma_{n+1}=\ldots=\sigma_{n+e}>\sigma_{n+e+1} \geq \ldots \geq \sigma_{n+d} \geq 0,
\end{gathered}
$$

and the corresponding partitioning

$$
V=\left[\begin{array}{l|l|l}
V_{11} & V_{12} & V_{13} \\
\hline V_{21} & V_{22} & V_{23}
\end{array}\right], \quad V_{11} \in \mathbb{R}^{d \times p}, V_{12} \in \mathbb{R}^{d \times(n-p+e)}, V_{13} \in \mathbb{R}^{d \times(d-e)} .
$$

The matrix $V_{12}$ represents the first $d$ rows of the block of singular vectors of $[B, A]$ corresponding to $\sigma_{n+1}$, while $V_{11}$ and $V_{13}$ represent the first $d$ rows of the block of singular vectors of $[B, A]$ corresponding to singular values strictly greater and strictly smaller than $\sigma_{n+1}$, respectively.

The classical analysis [4] of the TLS problem (2) with $d>1$ is based on the rank of the matrix $\left[V_{12}, V_{13}\right]$ and deals with three special cases. It is shown that if $\left[V_{12}, V_{13}\right]$ has full row rank $d$ and either $p=n$ (i.e. $\sigma_{n}>\sigma_{n+1}$ ) or $e=d$ (i.e. $\left.\sigma_{n+1}=\sigma_{n+d}\right)$, then a TLS solution exists. If $\operatorname{rank}\left(\left[V_{12}, V_{13}\right]\right)<d$ then the TLS solution does not exist, but the TLS concept is in [4] extended to the so called nongeneric solution. The case $\left[V_{12}, V_{13}\right]$ of full row rank $d$ with $p<n$ and $e<d$, is not analyzed in the literature known to us, and a TLS solution is not defined. The TLS algorithm [4, Algorithm 3.1, pp. 87-88] computes, however, some solution $X$ for any problem (1).

The analysis of the TLS problem in $[8,9]$ looks at individual ranks of the matrices $V_{12}$ and $V_{13}$. Let $\left[V_{12}, V_{13}\right]$ be of full row rank $d$. We can distinguish three cases which offer the classification of TLS problems for $d>1$ :

1. If $\operatorname{rank}\left(\left[V_{12}, V_{13}\right]\right)=d$ with $\operatorname{rank}\left(V_{12}\right)=e$ and $\operatorname{rank}\left(V_{13}\right)=d-e$ (maximal), then there exists a TLS solution of (1). If, moreover, $p=n$, then the solution is unique.

2. If $\operatorname{rank}\left(\left[V_{12}, V_{13}\right]\right)=d$ with $\operatorname{rank}\left(V_{12}\right)>e$ and $\operatorname{rank}\left(V_{13}\right)=d-e$ (maximal), then there exists a TLS solution of (1).

3. If $\operatorname{rank}\left(\left[V_{12}, V_{13}\right]\right)=d$ with $\operatorname{rank}\left(V_{12}\right)>e$ and $\operatorname{rank}\left(V_{13}\right)<d-e$, then the TLS solution of (1) does not exist.

It is worth to note that the TLS algorithm [4, Algorithm 3.1, pp. 87-88] computes a TLS solution, i.e. a solution satisfying (2), only in the case $\mathbf{1}$. The cases [ $V_{12}, V_{13}$ ] of full row rank $d$ with $p=n$ or $e=d$ analyzed in [4], are special cases of case 1 in our classification.

This classification may be completed by the fourth case:

4. If $\operatorname{rank}\left(\left[V_{12}, V_{13}\right]\right)<d$, then the TLS solution of (1) does not exist.

Note that since the classification of situations which can occur is complicated, we can not follow the basic, generic and nongeneric terminology used in [4]. Here we presented several ideas which initiated our work on the subject. Results of a joint effort with Diana M. Sima and Sabine Van Huffel focused on the analysis of TLS problems with multiple right-hand sides, will be presented elsewhere. Extension of the core reduction to problems with multiple right-hand sides is under development.

Acknowledgements This work was supported by the GAAV grant IAA100300802, and by the Institutional Research Plan AV0Z10300504. The research of I. Hnětynková was supported by the research project MSM0021620839 financed by MSMT.

\section{References}

[1] G. H. Golub, and W. Kahan, Calculating the singular values and pseudo-inverse of a matrix, SIAM J. Numer. Anal. Ser. B 2, pp. 205224 (1965).

[2] G. H. Golub, and C. F. Van Loan, An analysis of the total least squares problem, SIAM J. Numer. Anal. 17, pp. 883-893 (1980).

[3] I. Hnětynková, and Z. Strakoš, Lanczos tridiagonalization and core problems, Lin. Alg. Appl. 421, pp. 243-251 (2007).

[4] S. Van Huffel, and J. Vandewalle, The total least squares problem: computational aspects and analysis, (SIAM, Philadelphia, 1991).

[5] C. C. Paige, and Z. Strakoš, Scaled total least squares fundamentals, Numer. Math. 91, pp. 117-146 (2002).

[6] C. C. Paige, and Z. Strakoš, Unifying least squares, total least squares and data least squares, in "Total Least Squares and Errors-inVariables Modeling", S. van Huffel and P. Lemmerling, editors, Kluwer Academic Publishers, Dordrecht, pp. $25-34$ (2002).

[7] C. C. Paige, and Z. Strakoš, Core problems in linear algebraic systems, SIAM J. Matrix Anal. Appl. 27, pp. 861-875 (2006).

[8] M. Plešinger, The total least squares problem and reduction of data in $A X \approx B, \mathrm{PhD}$. thesis, Technical University of Liberec (2008).

[9] I. Hnětynková, M. Plešinger, D. M. Sima, Z. Strakoš, and S. Van Huffel, Analysis of the total least squares problem with multiple right-hand sides, in preparation. 